\title{
DEPRESSION, ANXIETY AND STRESS AMONG ADULTS IN PUTRAJAYA, MALAYSIA: A CROSS-SECTIONAL STUDY
}

\author{
Raudah Abd Rahman, Idayu Badilla Idris, Husnina Ibrahim \\ Department of Community Medicine, Faculty of Medicine, \\ University Kebangsaan Malaysia.
}

\begin{abstract}
Background: Mental health problem is rising globally, affecting the function and quality of life of the sufferers. In 1996, National Health Morbidity Survey (NHMS) showed that the prevalence of Malaysian aged 16 years and above with mental illness was $10.7 \%$ but in 2015, the prevalence had increased to $29.2 \%$. The aim of this study was to determine the prevalence of depression, anxiety and stress, and their associated sociodemographic and other factors.

Subjects and Method: This study was a cross-sectional study using data from the ongoing Mental Health Screening and Health Status Screening Programs among adults in Putrajaya, Malaysia. Data was obtained from 576 study subjects who attended the clinic via self-administered questionnaire. Mental Health Screening questionnaire which consisted of Depression Anxiety Stress Scale (DASS-21) and Health Status Screening questionnaire which consisted of medical history, smoking, alcohol consumption, drug abuse, history of violence, duration of exercise per week, and body mass index (BMI) was utilized in this study.

Results: The prevalence of depression, anxiety, and stress were $20.5 \%$, $31.6 \%$, and $10.1 \%$, respectively. Mean depression, anxiety, and stress score were significantly different among study subjects with history of being abused with $\mathrm{p}=0.006, \mathrm{p}=0.001$, and $\mathrm{p}=0.001$ respectively. Marital status was the only factor that was significantly associated with anxiety and stress score with $\mathrm{p}=0.025$ and 0.029 respectively, but was not significantly associated with depression score $(\mathrm{p}=0.088)$. Other factors were not statistically associated with depression, anxiety, and stress.

Conclusion: History of being abused and single was associated with the prevalence of depression, anxiety, and stress. However, further study is needed to investigate other significant factors of depression, anxiety, and stress for future intervention.
\end{abstract}

Keywords: depression, anxiety, stress, adults, Putrajaya

Correspondence: Idayu Badilla Idris. Department of Community Medicine Faculty of Medicine, University Kebangsaan Malaysia.

Email: idayubadilla.idris@gmail.com. 